\title{
Mediastinal Hemorrhage, CTCAE
}

National Cancer Institute

\section{Source}

National Cancer Institute. Mediastinal Hemorrhage, CT CAE. NCI Thesaurus. Code C56549.

A disorder characterized by bleeding from the mediastinum. 